\title{
Prevalencia de hipertrofia ventricular izquierda en pacientes hipertensos
}

\author{
Prevalence of left ventricular hypertrophy in \\ hypertensive patients
}

\author{
Fred Gustavo Manrique, Juan Manuel Ospina, \\ Giomar Maritza Herrera-Amaya • Tunja, Boyacá (Colombia)
}

\section{Resumen}

Antecedentes: se ha documentado a la hipertrofia ventricular izquierda como una de las manifestaciones tempranas de afectación cardiaca en la enfermedad hipertensiva.

Objetivo: evaluar la prevalencia de hipertrofia ventricular izquierda en los pacientes hipertensos que asisten a los programas de control en instituciones de salud de Boyacá.

Material y métodos: mediante muestreo secuencial aleatorio se ensambló una muestra de 1275 pacientes a quienes se realizó valoración de la presión arterial y electrocardiograma. Se evaluaron criterios de Cornell, Romhilt-Estest y Rodríguez-Padial para determinar la presencia de hipertrofia ventricular izquierda.

Resultados: se encontró prevalencia global de $17.9 \%$ de HVI en los pacientes analizados, con marcadas diferencias por municipio. La HVI se encontró asociada con edad mayor de 65 años, sexo femenino, índice de masa corporal aumentado y cifras elevadas de presión sistólica y diastólica.

Conclusión: estos resultados sugieren la necesidad de incrementar los programas de tamizaje y control de la presión arterial, así como de incluir capacitación a los agentes de salud en la valoración de evidencia sugestiva de HVI como alteraciones electrocardiográficas y diferencias en las presiones entre las dos extremidades superiores. (Acta Med Colomb 2014; 39: 244-249)

Palabras clave: hipertrofia ventricular izquierda, hipertensión arterial, factores de riesgo cardiovasculares, enfermedad cardiovascular, electrocardiografía.

\section{Abstract}

Background: left ventricular hypertrophy has been documented as one of the early manifestations of cardiac involvement in hypertensive disease.

Objectives: to assess the prevalence of left ventricular hypertrophy in hypertensive patients attending control programs in health institutions of Boyacá.

Materials and methods: by sequential random sampling, a sample of 1275 patients for whom assessment of blood pressure and electrocardiogram was performed, was assembled. Cornell, Romhilt-ESTest and Rodriguez-Padial criteria were evaluated to determine the presence of left ventricular hypertrophy.

Results: overall prevalence of LVH in the patients studied was $17.9 \%$, with marked differences respect to the village of origin. $\mathrm{LVH}$ was found associated with age over 65 , female gender, increased body mass index and high levels of systolic and diastolic pressure.

Conclusion: these results suggest the need for increased screening programs and control of blood pressure as well as include training to health workers in assessing suggestive evidence of LVH as electrocardiographic changes and differences in pressure between the two upper limbs. (Acta Med Colomb 2014; 39: 244-249)

Keywords: left ventricular hypertrophy, hypertension, cardiovascular risk factors, cardiovascular disease, electrocardiography.

Dr. Fred Gustavo Manrique: Profesor Titular Universidad Nacional de Colombia. Profesor Asociado UPTC- Grupo Salud Pública- CIECOL; Dr. Juan Manuel Ospina: Profesor Titular Escuela de Medicina, Universidad Pedagógica y Tecnológica de Colombia, Tunja U.P.T.C. Investigador Grupo de Investigación en Salud Pública GISP; Dra. Giomar Maritza Herrera-Amaya: Profesora Asistente Escuela de Enfermería, Universidad Pedagógica y Tecnológica de Colombia, Tunja U.P.T.C. Investigador Grupo de Investigación en Salud Pública GISP. Correspondencia. Dr. Juan Manuel OspinaDíaz. Tunja, Boyacá (Colombia). E-mail: juan.ospina@uptc.edu.co Recibido: 29/VII/2013 Aceptado: 1/VIII/2014 


\section{Introducción}

La hipertensión arterial (HTA) es una condición clínica asociada a trastornos en el metabolismo de lípidos y afectaciones endocrinas, que afecta alrededor de 1000 millones de personas en todo el orbe (1) y precursora del complejo sindromático de las enfermedades cardiovasculares (ECV), consideradas hasta el momento como la primera causa de muerte a nivel mundial, representando cerca de la mitad de todas las muertes ocasionadas por enfermedades crónicas no transmisibles en países de bajos y medianos ingresos. En la actualidad la estrategia central de prevención se centra en la identificación temprana e intervención sobre los factores de riesgo prevenibles (2).

Habida cuenta de que el corazón es uno de los órganos blanco afectados en la enfermedad hipertensiva, se considera que la hipertrofia ventricular izquierda (HVI) es la manifestación más frecuente de esta afectación y se ha caracterizado en general por aumento de peso del corazón, crecimiento del ventrículo izquierdo (VI), aumento del grosor parietal del VI, aumento del cociente del radio del VI respecto del grosor, aumento de la masa del VI y, a nivel microscópico, hipertrofia de los miocitos (3). Además de la hipertensión arterial, la HVI se asocia con la valvulopatía aórtica, ya sea insuficiencia o estenosis, cardiomiopatía hipertrófica y coartación de la aorta (4).

Debido a estas circunstancias se considera que la HVI constituye un predictor de mortalidad y morbilidad cardiovascular, en la actualidad algunos expertos consideran que el incremento en las prevalencias de HVI en algunas regiones como Norteamérica ha adquirido características de epidemia, por cuanto afecta alrededor de $16 \%$ de la población adulta. Además se reconoce que en estos pacientes se incrementa significativamente el riesgo de arritmias, enfermedad isquémica del corazón, infartos, falla cardiaca, etc. (5).

Diversos estudios han demostrado que la HVI es potencialmente reversible y que adecuadas medidas terapéuticas, concretamente las que normalicen el perfil lipídico y las que disminuyan las cifras tensionales, llevan a remodelación de la masa del miocardio optimizando la funcionalidad de los miocitos (6). Si bien es cierto que la HVI constituye un estadio en el proceso de la historia natural de la enfermedad hipertensiva, dado por la sobrecarga hemodinámica, que se traduce en fenómenos como el estiramiento de los miocitos, liberación de calcio intracelular, activación de la enzima calcineurina y la inducción de la reprogramación de expresión de los genes (7), la temprana identificación del fenómeno, es un criterio importante para la instauración o corrección de las medidas terapéuticas más adecuadas, en procura de mejorar el pronóstico y el estado de bienestar de los pacientes (8); de la misma manera, la prevalencia de HVI en pacientes de control de la HTA puede ser un buen indicador de la calidad y eficacia del correspondiente programa.

Es así como la prevalencia global varía significativamente de un área a otra; por ejemplo en China, con pacientes con media de edad 58.6 años, la prevalencia fue de $42.7 \%$ (9); en España de 18.5\% con edad media de 64 años (10); en Grecia 33.0\% con edad media de 61.9 años (11); en Nigeria $20.79 \%$ con edad media de 58-59 años (12).

En la actualidad, diversas técnicas de ecocardiografía constituyen la herramienta más confiable para el diagnóstico de la HVI; el problema radica en la reducida capacidad de acceso a esta tecnología en la mayoría de localidades de las provincias, particularmente en las pequeñas y medianas ciudades; por esta razón, se reconoce que el diagnóstico mediante electrocardiografía puede ser una herramienta útil para detectar precozmente esta complicación y adelantar intervenciones adecuadas y sobre todo, oportunas.

Se han propuesto diferentes algoritmos y esquemas de interpretación electrocardiográfica para hacer el diagnóstico de HVI; la mayoría de estos criterios considera la amplitud de las ondas del complejo Q y su duración; los principales modelos propuestos son los de Sokolow-Lion, Cornell, Romhil-Estes y de Rodríguez-Padial (13). En consideración a la baja especificidad y sensibilidad de la mayoría de estos criterios, tomados de manera aislada, se considera que la combinación de varios de ellos puede ser una alternativa que mejore razonablemente el rendimiento operativo de estos tests en el diagnóstico de la HVI. El propósito del presente estudio se focalizó en establecer la prevalencia de HVI en pacientes hipertensos adscritos a los programas de control de la HTA, y residentes en las áreas urbanas de nueve municipios del departamento de Boyacá, incluyendo los tres mayores: Tunja, Duitama y Sogamoso mediante el empleo de métodos electrocardiográficos validados.

\section{Material y métodos}

Se diseñó un estudio observacional, de corte transversal analítico. Como población general se definió a las personas hipertensas y como población objetivo a las personas hipertensas adscritas a los programas de control de la hipertensión arterial en las diferentes IPS públicas y privadas del departamento de Boyacá. La muestra se estimó acorde con una prevalencia de HVI en pacientes hipertensos, calculada en $12 \%$, acorde con un reporte reciente (14), y asumiendo efecto de diseño $=1$, con significancia de $95 \%$, para una estimación de mínimo 572 sujetos por incluir. Acorde con la resolución 8430/93 del Ministerio de Salud, el estudio fue aprobado por el comité de ética en investigación institucional y clasificado como de riesgo mínimo, por lo cual se solicitó a cada uno de los sujetos elegibles que diera lectura y expresara su acuerdo a participar voluntariamente signando un formato de consentimiento informado.

Se consideró elegibles a hombres y mujeres hipertensos, mayores de 40 años, con duración del complejo QRS inferior a $120 \mathrm{mseg}$. No se incluyeron pacientes con antecedente de enfermedad renal crónica, hipertiroidismo, valvulopatía, cardiomiopatía hipertrófica, bloqueos de rama o enfermedad de Chagas.

A todos los participantes se les aplicó, mediante entrevista, un cuestionario que incluía información sobre el 
paciente, su tratamiento y los factores de riesgo cardiovasculares a los que estaba expuesto, acorde con los criterios de Framingham. Posteriormente, y mediante protocolo previamente establecido se procedió a practicar medición de la presión arterial, valoración física y un electrocardiograma, garantizando condiciones de reposo y privacidad. Tanto las valoraciones como los electrocardiogramas se realizaron por profesionales de la salud previamente entrenados, siguiendo rigurosamente un protocolo establecido de antemano. La lectura de los electrocardiogramas se hizo por profesionales médicos; todos ellos se valoraron acorde con los criterios de Cornell, Romhilt-Estes y Rodríguez-Padial. Los resultados se clasificaron acorde con la evaluación separada de cuáles pacientes cumplían con uno, dos o los tres criterios de HVI; la estimación global de la prevalencia de punto de HVI se adelantó mediante la ponderación ajustada de los pacientes que cumplían con al menos uno de los criterios de HVI, los que cumplían dos y todos los que cumplían tres criterios.

El análisis univariado permitió examinar las características de cada uno de los dos grupos (con HVI y sin HVI), y determinar su comparabilidad en una línea de base. Cada variable de interés se describió según el nivel de medición; medias para las variables continuas, proporciones para las categóricas o nominales; todas se estimaron con los correspondientes intervalos de confianza (IC), en nivel alfa= 0.05. La comparación entre grupos se realizó a través de pruebas t de Student, ANOVA y de Chi cuadrado, cuando era pertinente.

Posteriormente, un análisis bivariado permitió establecer la asociación de las covariables con la variable de salida HVI. Esto fue realizado mediante el cálculo de medidas de efecto absoluto, Odds Ratio (OR) dada la exposición y sus respectivos IC al 95\%, junto con el cálculo de los valores p. Un análisis estratificado se realizó de manera exploratoria, con el fin de identificar posibles variables confusoras.

\section{Resultados}

Caracterización de la muestra. Mediante muestreo aleatorio secuencial por conveniencia, se evaluó un total de 1275 sujetos, residentes en nueve municipios del departamento de Boyacá. Del total de la muestra analizada el 74.8\% eran mujeres ( $\mathrm{n}=954)$. La edad media fue 67.5 años $(\mathrm{SD}=9.8)$. La media de edad de los hombres fue de $69.6(\mathrm{SD}=9.5)$ y de las mujeres $66.8(\mathrm{SD}=9.8)$, encontrándose diferencias estadísticamente significativas en el género $(\mathrm{p}<0.0001)$. La media de edad por municipio se muestra en la Tabla 1, 95.9\% de los encuestados pertenecía a los estratos socioeconómicos 1 y 2 mientras, que el $4.1 \%$ restante era de estrato 3 o superior $(n=52)$.

En $39.1 \%$ de las personas se encontró índice de masa corporal (IMC) superior a 25, es decir, se encuentra en sobrepeso u obesidad; a esta categoría pertenecen el $48.3 \%$ de las mujeres y $36.1 \%$ de los hombres. El $23.3 \%$ registra prevalencia de vida en el consumo de tabaco y $72.1 \%$ de alcohol. La mayor prevalencia en consumo de tabaco se encuentra en Sogamoso con $39.5 \%$, mientras que la mayor tasa de prevalencia de vida en el consumo de alcohol está en Duitama con $76.3 \%$.

La media de presión arterial sistólica fue 136.13 en hombres $(\mathrm{SD}=17.6)$ y 133.4 en mujeres $(\mathrm{SD}=17.05)$; se encontró diferencia significativa por género ( $\mathrm{p}$ de Kruskal - Wallis = 0.0067). La mayor media se registró en Tunja (138.83) y la menor en Motavita (130.56). La media de presión arterial sistólica en los pacientes con HVI fue de 138.16 (SD=18.17), mientras que en los sujetos sin HVI fue de 132.9 (SD=16.7); se encontró diferencia estadísticamente significativa entre los dos grupos $(\mathrm{p}<0.0001)$.

La media de presión arterial diastólica fue de 82.86 en hombres $(\mathrm{SD}=11.3)$ y de 81.97 en mujeres $(\mathrm{SD}=9.84)$; no se encontró diferencia significativa por género (p de Kruskal Wallis = 0.2536). La mayor media se registró en Ramiriquí (87.63) y la menor en Siachoque (80.15). En los pacientes con HVI la media de presión arterial diastólica fue de 85.27 $(\mathrm{SD}=11.8)$, mientras que en los pacientes sin HVI fue de 81.3 ( $\mathrm{SD}=9.5)$; se encontró diferencia estadísticamente significativa entre los dos grupos $(\mathrm{p}<0.0001)$.

Mediante la escala de apoyo social y estrés de Duke, se encontró déficit de apoyo social en $10.5 \%$ de los hipertensos y estrés en $48.7 \%$ de ellos.

Se registraron prevalencias de HVI con marcadas diferencias entre urbes medianas y poblaciones relativamente pequeñas, en un rango comprendido entre 4.9 y $52.7 \%$. La prevalencia global de HVI es similar a la reportada en estudios adelantados en otras latitudes $(15,16)$. Las prevalencias por municipio y global se muestran en la Tabla 2 .

Se encuentran en el análisis de prevalencias dos grupos de municipios: con prevalencias inferiores a las reportadas en estudios adelantados en otros países y otros con prevalencias superiores. Llama la atención el alto registro de Tunja, que contrasta significativamente con los de Duitama y Sogamoso, ya que son tres municipios mayores, con magnitudes poblacionales similares y sede de instituciones de tercer nivel.

Tabla 1. Media de edad por municipio de residencia. HTA-HVI en Boyacá. 2012.

\begin{tabular}{|l|c|c|c|}
\hline Municipio & Frecuencia & Media & Desv. estándar \\
\hline Duitama & 375 & 65.16 & 9.58 \\
Motavita & 53 & 68.05 & 8.37 \\
Ramiriquí & 55 & 67.94 & 6.76 \\
Samacá & 102 & 68.15 & 9.55 \\
Siachoque & 88 & 69.89 & 9.9 \\
Sogamoso & 223 & 69.74 & 9.43 \\
Soracá & 55 & 68.25 & 9.22 \\
Tóopaga & 80 & 65.86 & 11.13 \\
Tunja & 244 & 68.27 & 10.51 \\
\hline Total & 1275 & 67.5 & 9.84 \\
\hline
\end{tabular}


Tabla 2. Prevalencia de HVI por género y municipio. Boyacá 2012.

\begin{tabular}{|c|c|c|c|c|c|c|}
\hline \multirow[t]{2}{*}{ Municipio } & \multirow[t]{2}{*}{ HIV } & \multicolumn{2}{|c|}{ Género } & \multirow[t]{2}{*}{ Total } & \multirow[t]{2}{*}{ Prevalencia } & \multirow[t]{2}{*}{ IC95\% } \\
\hline & & Hombre (\%) & Mujer (\%) & & & \\
\hline \multirow[t]{2}{*}{ Tunja } & SÍ & $7(14.5)$ & $70(35.7)$ & 77 & 31.60 & $25.8-37.8$ \\
\hline & NO & 41 & 126 & 167 & 68.50 & $62.2-74.2$ \\
\hline \multirow[t]{2}{*}{ Sogamoso } & SÍ & $1(1.7)$ & $10(6.06)$ & 11 & 4.90 & $2.5-8.7$ \\
\hline & NO & 57 & 155 & 212 & 95.10 & $91.3-97.5$ \\
\hline \multirow[t]{2}{*}{ Duitama } & Sí & $3(3.26)$ & $19(6.71)$ & 22 & 5.86 & $3.8-8.9$ \\
\hline & NO & 89 & 264 & 353 & 94.14 & $91.1-96.2$ \\
\hline \multirow[t]{2}{*}{ Ramiriquí } & Sí & $2(25.0)$ & $27(58.69)$ & 29 & 52.70 & $38.8-66.3$ \\
\hline & NO & 6 & 20 & 27 & 47.30 & $33.7-61.2$ \\
\hline \multirow[t]{2}{*}{ Siachoque } & Sí & $10(32.2)$ & $13(22.8)$ & 23 & 27.05 & $17.3-36.6$ \\
\hline & NO & 21 & 44 & 65 & 72.95 & $63.4-82.7$ \\
\hline \multirow[t]{2}{*}{ Tópaga } & Sí & $6(27.3)$ & $6(10.34)$ & 12 & 15.00 & $8.0-24.7$ \\
\hline & NO & 16 & 52 & 68 & 85.00 & $75.3-92.0$ \\
\hline \multirow[t]{2}{*}{ Samacá } & Sí & $12(38.7)$ & $24(33.8)$ & 36 & 35.29 & $7.7-22.0$ \\
\hline & NO & 19 & 47 & 66 & 64.71 & $78.0-92.3$ \\
\hline \multirow[t]{2}{*}{ Motavita } & Sí & $7(43.7)$ & $10(27.0)$ & 17 & 32.07 & $18.5-45.5$ \\
\hline & NO & 9 & 27 & 36 & 67.93 & 54.4-81.4 \\
\hline \multirow[t]{2}{*}{ Soracá } & Sí & $0(0.0)$ & $2(5.0)$ & 2 & 3.63 & $0.44-12.5$ \\
\hline & NO & 15 & 38 & 53 & 96.37 & 87.4-99.5 \\
\hline \multirow[t]{2}{*}{ Total } & Sí & 48 (14.9) & 181 (18.9) & 229 & 17.96 & $13.01-17.06$ \\
\hline & NO & 273 & 773 & 1046 & 84.96 & 82.9-86.9 \\
\hline
\end{tabular}

La relación de la HVI con algunos factores se observa en la Tabla 3.

\section{Discusión}

La hipertensión arterial ha evolucionado en las últimas décadas afectando significativamente la calidad y expectativa de vida de cerca de $20 \%$ de las poblaciones urbanas en Colombia. Se estima que cifras elevadas de presión arterial incrementan hasta $90 \%$ el riesgo de desarrollar enfermedades cardiovasculares; de ahí el interés en reconocer rápidamente las manifestaciones de enfermedad hipertensiva (17). La HVI es con mucho, la afectación más común de daño a los órganos blanco en la enfermedad hipertensiva.

Diversos estudios han demostrado que la presencia de HVI incrementa el riesgo de enfermedad cerebrovascular, enfermedad coronaria, insuficiencia cardiaca congestiva, arritmias y muerte súbita de origen cardiaco; este riesgo se correlaciona significativamente con otros factores de riesgo cardiovascular reconocidos como la edad, el género, diabetes, obesidad y sobrepeso, inactividad física (18). En particular, en

Tabla 3. Factores posiblemente asociados con HVI en pacientes hipertensos. Boyacá 2012.

\begin{tabular}{|c|c|c|c|c|c|c|}
\hline \multirow{2}{*}{ Factor } & & \multicolumn{2}{|c|}{ Diagnóstico HVI } & \multirow{2}{*}{ OR } & \multirow{2}{*}{ IC95\% } & \multirow{2}{*}{$\mathbf{P}$} \\
\hline & & Sí & NO & & & \\
\hline Mayor 65 años & $\begin{array}{c}\text { Sí } \\
\text { NO }\end{array}$ & $\begin{array}{c}187 \\
98 \\
\end{array}$ & $\begin{array}{r}576 \\
414 \\
\end{array}$ & 1.36 & $1.04-1.8$ & 0.028 \\
\hline Género femenino & $\begin{array}{c}\text { Sí } \\
\text { NO }\end{array}$ & $\begin{array}{c}227 \\
58\end{array}$ & $\begin{array}{l}727 \\
263\end{array}$ & 1.41 & $1.02-1.94$ & 0.018 \\
\hline Prevalencia de vida tabaco & $\begin{array}{l}\text { Sí } \\
\text { NO }\end{array}$ & $\begin{array}{c}52 \\
233\end{array}$ & $\begin{array}{l}244 \\
746\end{array}$ & 0.68 & 0.49-0.95 & 0.013 \\
\hline Estrés & $\begin{array}{c}\text { Sí } \\
\text { NO }\end{array}$ & $\begin{array}{l}36 \\
22 \\
\end{array}$ & $\begin{array}{l}325 \\
358 \\
\end{array}$ & 1.80 & $1.03-3.12$ & 0.017 \\
\hline Presión diastólica >85 & $\begin{array}{l}\text { SÍ } \\
\text { NO }\end{array}$ & $\begin{array}{l}121 \\
164\end{array}$ & $\begin{array}{l}274 \\
714\end{array}$ & 1.92 & $1.46-2.52$ & $<0.001$ \\
\hline Presión sistólica $>140$ & $\begin{array}{l}\text { Sí } \\
\text { NO }\end{array}$ & $\begin{array}{c}86 \\
199\end{array}$ & $\begin{array}{l}207 \\
781\end{array}$ & 1.63 & $1.21-2.19$ & 0.0009 \\
\hline $\mathrm{IMC}>25$ & $\begin{array}{l}\text { Sí } \\
\text { NO }\end{array}$ & $\begin{array}{l}126 \\
159\end{array}$ & $\begin{array}{l}373 \\
617\end{array}$ & 1.31 & $1.003-1.711$ & 0.027 \\
\hline
\end{tabular}


nuestro medio, la diabetes reviste particular importancia dada su alta prevalencia y la asociación demostrada con HVI (19).

Si bien la prevalencia global de HVI en pacientes hipertensos, con edades similares a las reportadas, es concordante con lo reportado en otros estudios adelantados en Iberoamérica, la marcada diferencia encontrada entre municipios que revisten cierto grado de homogeneidad poblacional, socioeconómica y en la capacidad instalada para ofertar servicios de salud es el hallazgo más llamativo, ya que probablemente se relaciona tanto con la calidad de los servicios de salud como con los estilos de vida y con el probable sesgo atribuible a la técnica de diagnóstico empleada en el estudio, definitivamente menos confiable que el ecocardiograma, pero más accesible (20).

También es importante considerar la necesidad de involucrar en el entrenamiento de los profesionales de la salud la valoración de aspectos integrales no sólo del electrocardiograma, sino en la valoración física. Tal es el caso de la detección de las diferencias en la presión sanguínea entre los dos brazos, signo de muy fácil realización, que también puede orientar hacia el diagnóstico de HVI, pero que lamentablemente no está considerado en la rutina de los protocolos de control de pacientes hipertensos en los controles de la consulta médica institucional (21).

De todas maneras, el reconocimiento de la prevalencia de HVI en pacientes hipertensos consolida información que es muy útil al establecer la necesidad de reestructurar los protocolos de diagnóstico y manejo de este tipo de pacientes, dado que se reconoce la relación de la HVI con mayores tasas de mortalidad y morbilidad en los pacientes hipertensos (22).

En el presente estudio también se ha corroborado la relación existente entre la HVI y algunos factores no modificables como el género y la edad, ya que se encontró mayor riesgo en las mujeres y en los mayores de 65 años, lo cual se ha reportado también en estudios adelantados en otras regiones; de la misma manera, estos resultados concuerdan en identificar la asociación con otros factores de riesgo modificables como el índice de masa corporal, el consumo de alcohol, y cifras elevadas de las presiones sistólica y diastólica (23). Si bien en esta investigación no se evaluó el perfil lipídico, es importante anotar que alteraciones de los niveles séricos de colesterol y triglicéridos también se han reportado en la literatura asociados con la HVI.

Concomitantemente, se puede deducir que uno de los aspectos relevantes de estos resultados tiene que ver con la demostración clara de que la determinación temprana, mediante evidencia subclínica, del daño de un órgano blanco puede llevar a modificar el manejo de los pacientes y a intervenir sobre los riesgos para revertir la condición fisiopatológica mejorando así el pronóstico a mediano y largo plazo; este es el enfoque de riesgo que hace valioso el concepto de medicina preventiva (24).

Desde la perspectiva del costo-beneficio, se debe considerar que las prevalencias elevadas de HVI que se registran en algunos escenarios del estudio, y que contrastan significativamente con otras ciudades de similares características, llevan a pensar en la necesidad de mejorar los sistemas y protocolos de los programas de tamizaje y control de la HTA, por cuanto el diagnóstico general de la situación de salud puede ser el primer paso para optimizar los resultados esperados con las intervenciones y estrategias diagnósticas por implementar (25).

Se sugiere que los pacientes con trazados electrocardiográficos positivos para HVI sean remitidos para valoraciones específicas que incluyan ecocardiograma confirmatorio y control que permitan manejo para minimizar el riesgo de desarrollar cardiomiopatía dilatada, crecimiento atrial y fibrilación atrial en el futuro,

Es importante que en los programas de tamizaje y control se introduzca el concepto de comorbilidad, ya que es evidente que las consecuencias fisiopatológicas derivadas de los cambiantes estilos de vida inciden significativamente en el incremento poblacional de casos de hipertensión arterial, y consecuentemente, se incrementan significativamente los riesgos de enfermedad cardiovascular y muerte. Las estrategias de capacitación continua del personal de salud y de educación en salud deben replantearse y priorizarse con miras al abordaje de la creciente epidemia de obesidad y sobrepeso que se avecina en los países de medianos y bajos ingresos (26).

\section{Conclusiones}

El empleo de métodos electrocardiográficos con criterios estandarizados permitió establecer una prevalencia global de $17.96 \%$ de HVI en la población estudiada, con valores cambiantes entre localidades en un rango de 4.90-52.7\%,que al parecer refleja diferencias en la calidad y eficacia de los programas de control, fomento y prevención de la HTA en las diferentes ciudades.

La HVI se encontró asociada con la edad mayor de 65 años, género femenino, índice de masa corporal aumentado, valores de presión arterial sistólica y diastólica aumentados.

Se hace necesario que las instituciones cuenten con electrocardiógrafos para el control del paciente hipertenso en el primer nivel de atención y que el personal de salud reciba entrenamiento en la toma, e interpretación del mismo facilitando la detección temprana de cambios en el proceso de manejo de la HTA.

Ninguno declarado

\section{Conflicto de interés}

\section{Agradecimientos}

Los autores hacen expresa manifestación de agradecimiento a los profesionales en fermeros y médicos que participaron en el proceso de captura de datos y análisis de los registros electrocardiográficos; a la Dirección de Investigaciones de la U.P.T.C. por el apoyo logístico.

\section{Referencias}

1. Rivera Maza M,Suárez Osorio A. Dispersión de QT corregido para identificación de hipertrofia ventricular izquierda en pacientes con hipertensión arterial sistémica: Una serie de casos [Tesis Doctoral]. San salvador: Universidad Dr. Jose Matías Delgado; 2012. 
2. Otgontuya D, Oum S, Buckley B, Bonita R. Assessment of total cardiovascular risk using WHO/ISH risk prediction charts in three low and middle income countries in Asia. BMC Public Health 2013; 13 (1): 539.

3. Alpert M, Chan E. Morfología ventricular izquierda y función diastólica en la obesidad grave: perspectivas actuales (Editorial). Rev Esp Cardiol 2012; 65 (1): $1-3$.

4. Uribe W, Duque M, Medina E. Electrocardiografía y arritmias. Clínica Medellín. Editorial PLA Export Bogotá DC. 2005: 41-5

5. Bacchus R, Singh K, Ogeer I, Mungrue K. The occurrence of left ventricular hypertrophy in normotensive individuals in a community setting in North-East Trinidad. Vasc Health Risk Manag 2011; 7: 327-32.

6. Ferreira C, De Abreu L, Valenti V, Ferreira M, Meneghini A, Silveira J. Antihypertensive drugs have different effects on ventricular hypertrophy regression. Clinics 2010; 65 (7): 723-28.

7. Duarte D, Minicucci M, Azevedo P, Matsubara B, Matsubara L, Novelli E. The role of oxidative stress and lipid peroxidation in ventricular remodeling induced by tobacco smoke exposure after myocardial infarction. Clinics 2009; 64 (7): 691-7.

8. Rodríguez-Padial L, Rodríguez B, Jérez M, Casares J, Akerström F, Calderón A. Precisión diagnóstica del electrocardiograma asistido por ordenador al diagnosticar hipertrofia ventricular izquierda en el bloqueo de rama izquierda. Rev Esp Cardiol 2012; 65 (1): 38-46.

9. Wang S-x, Xue H, Zou Y-b, SUN K, FU C-y, WANG H, et al. Prevalence and risk factors for left ventricular hypertrophy and left ventricular geometric abnormality in the patients with hypertension among Han Chinese. Chinese Medical Journal 2012; 125 (1): 21-6.

10. De la Sierra A, Segura J, Banegas JR, Gorostidi M, Juan J, Armario P, et al. Clinical features of 8295 patients with resistant hypertension classified on the basis of ambulatory blood pressure monitoring. Hypertension 2011; 57 (5): 898-902.

11. Papazafiropoulou A, Skliros E, Sotiropoulos A, Papafragos C. Prevalence of target organ damage in hypertensive subjects attending primary care: C.V.P.C. study (epidemiological cardio-vascular study in primary care). BMC Family Practice 2011; 12 (75).

12. Salako BL, Ogah OS, Adebiyi AA, Oladapo OO, Aje A, Adebayo AK, et al. Blood pressure control and left ventricular hypertrophy in hypertensive Nigerians. Ann Afr Med 2009; 8 (3): 156-62.

13. Cabezas M, Comellas A, Gómez J, López L, Casal H, Carrillo N, et al. Comparación de la sensibilidad y especificidad de los criterios electrocardiográficos para la hipertrofia ventricular izquierda según métodos de Romhilt-Estes, SokolowLyon, Cornell y Rodríguez Padial. Rev Esp Cardiol 1997; 50 (1): 31-5.

14. Alvarado C, Jaramillo M, Matijasevic E, Rendón I, Quitian J. Estudio po- blacional de factores de riesgo cardiovascular relacionados con el estilo de vida, hallazgos electrocardiográficos y medicación actual de pacientes valorados por el servicio de Cardiología. Rev Colomb Cardiol 2012; 19 (2): 61-71.

15. Lozano J, Redon J, Cea-Calvo L, Fernández C, Navarro J, Bonet A, et al. Hipertrofia ventricular izquierda en la población hipertensa española. Estudio ERIC-HTA. Rev Esp Cardiol 2006; 59 (2): 136-42.

16. González J, Cea-Calvo L, Bertomeu V, Aznar J. Criterios electrocardiográficos de Hipertrofia ventricular izquierda y perfil de riesgo cardiovascular en hipertensos. Estudio VIIDA. Rev Esp Cardiol. 2007; 60 (2): 148-56.

17. Páez-Esteban A. Factores asociados al control de la hipertensión arterial en la población de hipertensos de los estratos 2 y 3 de Bucaramanga [Tesis de Maestría en Epidemiología]. Bucaramanga: Universidad Industrial de Santander; 2011.

18. Xue H, Fu Z, Chen Y, Xing Y, Liu J, Zhu H, et al. The Association of Growth Differentiation Factor-15 with Left Ventricular Hypertrophy in Hypertensive Patients. PLOS ONE On-Line. 2012; 7 (10).

19. Valarezo-Sevilla D, Pazmiño-Martínez A, Morales-Mora N. Prevalencia de hipertrofia ventricular izquierda en pacientes diabéticos. Rev Peru Med Exp Salud Publica 2013; 30 (1): 69-72.

20. Rosa E, Nunes D, Wilhelms R. Questioning the prevalence of left ventricular hypertrophy considered as reference in the study by its authors. Arq Bras Cardiol 2013; 100 (4): 386.

21. HM S, TH L, PC H, CY C, WH L, SC C. Association of Interarm Systolic Blood Pressure Difference with Atherosclerosis and Left Ventricular Hypertrophy. PLoS ONE On-line. 2012; 7 (8).

22. Bouzas A, Broullon F, Álvarez N, Peteiro J, Mosquera V, Castro A. Association of left ventricular mass with all-cause mortality, myocardial infarction and stroke. PLoS ONE (On-line). 2013; 7 (9).

23. Zhang Y, Li Y, Liu M, Sheng C-S, Huang Q-F, Wang J-G. Cardiac structure and function in relation to cardiovascular risk factors in Chinese. BMC cardiovascular disorders 2012; 12 (1): 86.

24. Desai C, Ning H, Lloyd-Jones D. Competing cardiovascular outcomes associated with electrocardiographic left ventricular hypertrophy: the Atherosclerosis Risk in Communities study. Heart 2012; 98 (4): 330-34.

25. Ortiz H, Vaamonde R, Zorrilla B, Arrieta F, Casado M, Medrano M. Prevalencia, grado de control y tratamiento de la hipertensión arterial en la población de 30 a 74 años de la comunidad de Madrid. Estudio Predimerc. Rev Esp Salud Pública 2011; 85 (4): 329-38.

26. Van der Berg N, Meinke-Franze C, Fiss T, Baumeister S, Hoffmann W. Prevalence and determinants of controlled hypertension in a German population cohort. . BMC Public Health 2013; 13: 594. 